\title{
UPLC-MS/MS quantification of quercetin in plasma and urine following parenteral administration
}

\author{
Igor A. Zupanets ${ }^{1 *}$ (D), Yuri V. Pidpruzhnykov ${ }^{1,2}$, Valerii E. Sabko², Natalia P. Bezugla ${ }^{1}$ and Sergii K. Shebeko ${ }^{1}$
}

\begin{abstract}
Background: Study of pharmacokinetics of quercetin $(\mathrm{Qu})$ and its derivatives after infusion is required in order to better understand mechanisms of therapeutic action of the formulation and development the strategic approaches to treat the underlying disease and pathologically similar syndromes. A pharmacokinetic study of Qu products is a complex analytical problem.

Results: We developed and validated a new method for quantification of Qu and its metabolites in human plasma and urine following intravenous administration of Qu formulation. The method is based on the solid-phase extraction with the help of Oasis ${ }^{\circledast}$ HLB cartridges and ultra-performance liquid chromatography - tandem mass spectrometry (UPLCMS/MS) quantification. The calibration curves plotted for concentration range of 25-3000 ng/ml for Qu and isorhamnetin in blood plasma and of 100-8000 $\mathrm{ng} / \mathrm{ml}$ for the same substances in urine were best described by a quadratic function. Qu conjugates underwent preliminary hydrolysis by the mixture of enzymes of sulphatase and $\beta$-glucuronidase. The antioxidant L-cysteine mixed with ascorbic acid was used for stabilization of Qu and its metabolites during handling of samples and chromatographic runs with mass detection. The method validation as well as the stability study results confirmed that the developed method meets the established requirements (selectivity, lower limit of quantification, accuracy, precision, recovery).

The main pharmacokinetic parameters of Qu and its metabolites were determined in blood plasma and urine after intravenous administration of Corvitin ${ }^{\circledast}$ (parenteral formulation combining Qu and polyvinylpyrrolidone at a ratio of 1:9) in 12 healthy volunteers.
\end{abstract}

Conclusions: We showed that the method development for Qu quantification in biological matrixes is valuable and reliable.

Keywords: Quercetin, Metabolites, UPLC-MS/MS, Quantification, Pharmacokinetics

\section{Introduction}

Quercetin [3,3',4',5,7-pentahydroxyflavone] is a natural bioflavonoid. Oral medical Qu products and dietary supplements containing $\mathrm{Qu}$ are used in medical practice, and their pharmacokinetic studies are described [1]. Qu parenteral formulations currently are not widely used, which, most probably, is related to poor solubility of $\mathrm{Qu}$. The original medical Qu formulation Corvitin manufactured by SIC Borshchahivskiy Chemical-Pharmaceutical Plant CJSC (Ukraine) is a combination of Qu with polyvinylpyrrolidone

\footnotetext{
*Correspondence: igorzupanets@gmail.com

'National University of Pharmacy, 27 Pushkinska Street, Kharkiv 61057,

Ukraine

Full list of author information is available at the end of the article
}

at a ratio of 1:9. The formulation is produced as a porous lyophilized powder of $500 \mathrm{mg}$ in a sterile vial and administered by intravenous infusion in physiological saline solution. Corvitin is used as a cardioprotector in therapy of acute coronary circulation failure and myocardial infarction, in therapy and prevention of reperfusion syndrome in surgery of patients with obliterating atherosclerosis of abdominal aorta and peripheral arteries. Pharmacokinetics study of $\mathrm{Qu}$ and its derivatives after infusion is required in order to better understand mechanisms of therapeutic action of the formulation and development the strategic approaches to treat the underlying disease and pathologically similar syndromes. 
A pharmacokinetic study of Qu products is a complex analytical problem. $\mathrm{Qu}$ is an easily oxidized substance, especially in neutral and alkaline media, thus, it is necessary to use effective antioxidants for preparing samples and performing further analysis. Moreover, $\mathrm{Qu}$ is rapidly metabolized in the body to methyl derivatives, various glucuronic and sulphate conjugates and their various combinations [2-5].

$\mathrm{Qu}$ and its metabolites are characterised by pronounced pharmacological activity [6-10], thus, in pharmacokinetic studies it is necessary to determine both free $\mathrm{Qu}$ and its metabolites. For quantitative determination of conjugated $\mathrm{Qu}$ in the form of glucuronide and sulphate derivatives, the method of enzymatic deconjugation is preferred [11]. At this point it is necessary to take into account that a part of $\mathrm{Qu}$ is metabolised to the form of methyl derivatives, and especially 3 '-O-methylquercetin (isorhamnetin, Ir), 4'-O-methylquercetin (tamarixetin) and their conjugates [12]. That is why during a bioavailability study of $\mathrm{Qu}$ products it is required to quantify the deconjugated methyl derivatives of $\mathrm{Qu}$, which is difficult in vitro because of reverse conversion to $\mathrm{Qu}$ by fermentation. A pharmacokinetic study of three doses of an oral Qu product, where it was assayed in healthy volunteers' blood plasma by using HPLC with a fluorescent radiation detector is described in [13], and meanwhile both sulphate and glucuronide conjugates of $\mathrm{Qu}$, and its methyl metabolites and their conjugates were determined.

The use of HPLC-MS/MS to determine $\mathrm{Qu}$ and its conjugates in blood plasma and urine after oral administration is described in [1]. Bioavailability of $\mathrm{Qu}$ and rutin after oral and parenteral administration in rats was studied in [14]. Both pharmacokinetics of free $\mathrm{Qu}$ and its conjugates, but not methyl derivatives of $\mathrm{Qu}$ and their conjugates, were determined in these two studies.

The first study of $\mathrm{Qu}$ pharmacokinetics after intravenous administration at a dose of $100 \mathrm{mg}$ with ethanol vehicle was performed in 1975 [15], however, selective methods (HPLC) for quantitative determination of $\mathrm{Qu}$ were not used. According with [16], Qu concentrations in blood plasma and urine after intravenous administration of $\mathrm{Qu}$ dissolved in water-dimethylsulfoxide (1:1) mixture was determined by HPLC using UV-detection, and at that point only free $\mathrm{Qu}$ (non-conjugated) was assayed. In this study, the solution of $\mathrm{Qu}$ in the mixture of water-dimethylsulfoxide was obtained immediately before administration, i.e. 'ex tempore'. Dimethylsulfoxide affects not only the solubility of $\mathrm{Qu}$, but also its bioavailability, and administration of $\mathrm{Qu}$ products in the form of water-dimethylsulfoxide solution results in adverse reactions in patients [16], and that, in all likelihood, was the reason for not introducing the investigated drug to market after the study.
In order to carry out the scheduled study, the method of ultra performance liquid chromatography (UPLC) with tandem MS/MS detection was used. This article describes the development and validation of a UPLC-MS/MS method for quantitative determination of $\mathrm{Qu}$ and its metabolites in blood plasma and urine of healthy volunteers. We believe that we are the first ones to perform a pharmacokinetic study of the commercial $\mathrm{Qu}$ formulation for intravenous administration currently used in medical practice.

\section{Materials and methods}

\section{Reagents, materials and solvents}

Quercetin dihydrate (100.0\% purity) and internal standard (IS) fisetin (99.0\% purity) were obtained from Sigma, isorhamnetin (99.6\% purity) - from Fluka, powder of the mixture of enzymes of sulphatase and $\beta$-glucuronidase (source - Helix pomatia), type $\mathrm{H}-1 \quad\left(\mathrm{~A}_{\mathrm{S}}=20,560\right.$ units/g; $\mathrm{A}_{\mathrm{G}}=2,701,900$ units/g) was obtained from Sigma, Corvitin ${ }^{\circ}$, lyophilized powder for injection, $0,5 \mathrm{~g}$ in vials was provided by SIC Borshchahivskiy Chemical-Pharmaceutical Plant CJSC (Ukraine), batch \# 1280408.

Methanol and acetonitrile (AcN) both 99.9\% of HPLC-grade were obtained from LAB-SCAN, formic acid $98-100 \%$, puriss. p.a. from Riedel-de-Haen.

All other chemicals were of analytical grade.

Water was purified by Elix-35 (Millipore, USA) with final purification using Milli-Q Gradient (Millipore, USA).

Oasis ${ }^{\circ}$ HLB 96 well cartridges, $10 \mathrm{mg}$ packing, grain size - $30 \mu \mathrm{m}$ (Waters, Milford, MA, USA) also were used.

Drug-free human plasma from healthy volunteers was provided by JCC Biofarma, Ukraine, each batch was accompanied by a certificate of analysis and was stored at $-30{ }^{\circ} \mathrm{C}$ prior to use.

Chromatography was performed in gradient mode. Solvent A: AcN - water - formic acid (10: 90: 0.2, v/v/ $\mathrm{v})$, solvent B: AcN - formic acid (100: 0.2, v/v).

\section{Instrumentation and methods}

The LC-MS/MS analyses were performed using a Waters Acquity UPLC ${ }^{\bullet}$ system with a Waters ACQUITY UPLC $^{\ominus}$ BEH C18 columns $(50 \mathrm{~mm} \times 2.1 \mathrm{~mm}, 1.7 \mu \mathrm{m})$ (both Milford, MA, USA) in combination with a Quattro Micro API triple quadrupole mass spectrometer (Micromass, Manchester, UK) equipped with an electrospray interface. MassLynx 4.1 software was used for instruments control and data processing.

Chromatography was performed at $40^{\circ} \mathrm{C}$ at a flow rate of $0.4 \mathrm{ml} / \mathrm{min}$ with a total run time of $1.4 \mathrm{~min}$. The start mobile phase consisted of $78 \%$ solvent $\mathrm{A}$ and $22 \%$ solvent $\mathrm{B}$ and the linear gradient run over $1 \mathrm{~min}$ up to $46 \%$ A, $54 \%$ B. Then composition was set immediately at $100 \% \mathrm{~B}$ and held constant $0.15 \mathrm{~min}$, followed back to $22 \%$ B. The autosampler was kept at $5{ }^{\circ} \mathrm{C}$ and $40 \mu \mathrm{l}$ samples were injected. 
Data acquisition was performed in the positive ion mode. The capillary voltage was set at $2000 \mathrm{~V}$, the source temperature was set at $100^{\circ} \mathrm{C}$ and the desolvation temperature was set at $500^{\circ} \mathrm{C}$. The desolvation gas flow (nitrogen, $99.5 \%$ ) was set at $750 \mathrm{~L} / \mathrm{Hr}$, the collision gas pressure (argon, 99.99\%) was set at $3.5 \mu \mathrm{bar}$.

Detection of the ions was performed in the multiple reaction monitoring mode (MRM), monitoring the ion combinations of $\mathrm{m} / \mathrm{z} 302.8 \rightarrow 152.7 \mathrm{amu}$ for $\mathrm{Qu}, 316.8$ $\rightarrow 152.7 \mathrm{amu}$ for Ir and $286.9 \rightarrow 136.7 \mathrm{amu}$ for fisetin (Fs). The collision energy was set at $34,40,30 \mathrm{~V}$ and the cone voltage was set at $45,40,45 \mathrm{~V}$ for $\mathrm{Qu}$, Ir and Fs respectively. These MRM-method parameters were determined by using Autotune option.

\section{Sample preparation}

\section{Preparation of standard and QC solutions}

A reference standards of $\mathrm{Qu}$ or Fs (about $5 \mathrm{mg}$ ) or $\mathrm{Ir}$ (about $2.5 \mathrm{mg}$ ) were accurately weighed, put into a $10 \mathrm{ml}$ volumetric flask, dissolved in ethanol containing $0.01 \%$ of butylhydroxytoluene in an ultrasonic bath while heating up to $50^{\circ} \mathrm{C}$ (stock solution). Shelf life of Qu and $\mathrm{Ir}$ solutions was 3 months, for Fs solution shelf life was at least 22 days when kept at +2 to $+7^{\circ} \mathrm{C}$.

The solution of the $\mathrm{Qu}$ and Ir mixture for calibration solutions were prepared by mixing and appropriate dilution of stock solutions by using water-AcN (3:1) mixture containing $0.005 \%$ ascorbic acid and $0.2 \%$ L-cysteine as solvent. Meanwhile $\mathrm{Qu}$ and Ir mixture solutions were obtained with the following concentrations of each analyte: 500; 1000; 1500; 2000; 3000; 5000; 10,000; 15,000; 20,$000 ; 40,000 ; 60,000 \mathrm{ng} / \mathrm{ml}$. An Fs solution (subsequently was used as IS) with a concentration of $8000 \mathrm{ng} /$ $\mathrm{ml}$ was prepared from the Fs stock solution by diluting with the abovementioned solvent. The solutions were kept at a temperature +2 to $+7{ }^{\circ} \mathrm{C}$.

The calibration solutions and QC solutions based on the respective blank plasma or urine were prepared by adding $23 \mathrm{ml}$ of the antioxidant solution (aqueous solution containing 10\% ascorbic acid, 4\% L-cysteine and 2\% formic acid), $20 \mu \mathrm{l}$ of respective solution of the Qu and Ir mixture and $40 \mu \mathrm{l}$ of the IS solution to $400 \mu \mathrm{l}$ of plasma or $100 \mu \mathrm{l}$ of urine mixed with $300 \mu \mathrm{l}$ of sodium-citrate buffer solution pH 5.0 in Eppendorf tubes $(1.5 \mathrm{ml})$. The solutions were vortexed and then the samples were prepared as described in Section 'Preparation of biological samples', beginning with adding $800 \mu \mathrm{l}$ of cooled $0.5 \mathrm{M}$ solution of $\mathrm{H}_{3} \mathrm{PO}_{4}$, containing $1 \%$ ascorbic acid.

As a result, the calibration solutions containing $\mathrm{Qu}$ and Ir analytes in concentrations of 25; 50; 100; 250; 500; 1000; 2000; $3000 \mathrm{ng} / \mathrm{ml}$ and QC solutions of the same analytes in concentrations of 75; 150; 750; 2000 $\mathrm{ng} / \mathrm{ml}$, calculated with reference to the content in plasma, as well as the calibration solutions containing
$\mathrm{Qu}$ and Ir analytes in concentrations of 100; 200; 400; 1000; 2000; 4000; $8000 \mathrm{ng} / \mathrm{ml}$ and QC solutions of the same analytes in concentrations of 300; 3000; $6000 \mathrm{ng} /$ $\mathrm{ml}$ calculated with the reference to the content in urine, were obtained. The Fs IS concentration in all the solutions based on plasma was $800 \mathrm{ng} / \mathrm{ml}$, and in those based on the urine was $3200 \mathrm{ng} / \mathrm{ml}$, with the reference to a corresponding biological matrix.

\section{Preparation of biological samples}

Two equal aliquots were sampled from fresh thawed biological samples; one of them was subjected to enzymatic deconjugation; at the same time, the second aliquot was kept in a freezer at a temperature no higher than $35^{\circ} \mathrm{C} .30 \mathrm{~min}$ before the end of enzymatic deconjugation, the frozen aliquots were thawed at room temperature. All the biological samples for analytical run, calibration and QC solutions were simultaneously treated.

In order to analyse routine plasma samples, two $400 \mu \mathrm{l}$ aliquots were sampled; to analyse urine samples, two $100 \mu \mathrm{l}$ aliquots were sampled; those were placed into Eppendorf tubes $(1.5 \mathrm{ml}) .300 \mu \mathrm{l}$ of sodium-citrate buffer solution $\mathrm{pH} 5.0$ were added to the urine samples and solutions were vortexed. $23 \mathrm{ml}$ of the abovementioned solution of antioxidants and $40 \mu \mathrm{l}$ of the respective IS solution were added to all the solutions. The solutions were vortexed.

To each test tube with a sample for enzymatic deconjugation the $10 \mu$ lof $5 \%$ solution of enzyme $\mathrm{F} \mathrm{H}-1$ in sodium-citrate buffer solution $\mathrm{pH} 5.0$ were added, and the solution was vortexed. Incubation was performed in a water bath at $(+37 \pm 1)^{\circ} \mathrm{C}$ for $17 \pm 0.1 \mathrm{~h}$. Immediately after incubation $800 \mu \mathrm{l}$ of cooled to 0 to $+4{ }^{\circ} \mathrm{C} 0.5 \mathrm{M}$ solution of $\mathrm{H}_{3} \mathrm{PO}_{4}$, containing $1 \%$ of ascorbic acid was added to these samples, as well as to the second part of the samples, which were kept in a freezer during the incubation and thawed before examination, and the solutions were vortexed.

The tubes with the samples were centrifuged at $28600 \mathrm{~g}$ for $10 \mathrm{~min}$ (the centrifuge was pre-cooled to $10^{\circ} \mathrm{C}$ ). The content of each tube was transferred by an automatic pipette to a respective cell of an Oasis ${ }^{\circ}$ HLB cartridge prewashed with $500 \mu \mathrm{l}$ of $0.5 \mathrm{M}$ solution of $\mathrm{H}_{3} \mathrm{PO}_{4}$ and, by creating "slight" vacuum, the solution was passed through the sorbent at a rate of about $0.2 \mathrm{ml} / \mathrm{min}$. $500 \mu \mathrm{l}$ of $5 \%$ solution of methanol in $0.5 \mathrm{M} \mathrm{H}_{3} \mathrm{PO}_{4}$ was added with the help of the automatic pipette to the same cells, the cells were kept for 1 min and drained under "slight" vacuum. Then $200 \mu \mathrm{l}$ of $50 \%$ solution of methanol in $0.5 \mathrm{M} \mathrm{H}_{3} \mathrm{PO}_{4}$ was added to the same cells by the automatic pipette, the cells were kept for $1 \mathrm{~min}$ and drained under vacuum till complete exsiccation of the sorbent. In the apparatus for vacuum filtration, the collection tray for washing solutions was replaced with a collector dish. Into each used cell of the Oasis ${ }^{\oplus}$ HLB 
cartridge, $300 \mu \mathrm{l}$ of methanol was poured, and they were kept for $1 \mathrm{~min}$ and then eluted into the collector dish, creating "slight" vacuum. The elution procedure was repeated one more time under the above conditions. To each used cell of the collector dish, $800 \mu$ of water solutions of ascorbic acid, L-cysteine and formic acid with concentrations of $0.005,0.002$, and $0.2 \%$, respectively, were added. The solutions in the cells were stirred by pipetting, and the air-tightly closed dish was taken for recording chromatograms.

\section{Method validation}

The validation of the developed method for determination of $\mathrm{Qu}$ and $\mathrm{Ir}$, as well as its sulphate and glucuronide conjugates in blood plasma and urine was performed in accordance with [17]. The validation covered the following parameters: selectivity, lower limit of quantification, calibration curve, within-run and between-run precision and accuracy, recovery, dilution procedure of biological samples of blood plasma, and stability.

In order to determine the selectivity, we used three different batches of respective blank biological matrices added with $\mathrm{Qu}$ and $\mathrm{Ir}$ in amounts corresponding to the LLOQ $(25 \mathrm{ng} / \mathrm{ml}$ calculated with the reference to the content in plasma or $100 \mathrm{ng} / \mathrm{ml}$ calculated with the reference to the content in urine), and IS at concentrations that were mentioned above in Section 'Preparation of standard and QC solutions'.

In order to determine the lower limit of quantification (LLOQ), we prepared 5 different solutions based on respective blank biological matrices added with $\mathrm{Qu}$ and Ir in amounts corresponding to the LLOQ and Fs IS, obtaining Fs concentrations of $800 \mathrm{ng} / \mathrm{ml}$ in plasma, and $3200 \mathrm{ng} / \mathrm{ml}$ in urine. The chromatograms of the obtained solutions, as well as solutions of blank plasma and urine, prepared as described above, were recorded and the peak areas in the respective chromatograms were compared.

In order to determine parameters of the calibration curve, while determining analytes in plasma and urine, respectively 8 and 7 calibration solutions with concentrations specified in Section 'Preparation of standard and QC solutions' were used.

In order to assess the parameters Precision and Accuracy five QC-solutions of each concentration were prepared; meanwhile four concentrations in plasma and three concentrations in urine were used (see Section 'Preparation of standard and QC solutions'). The experiment was carried out on three separate occasions. Within-run and between-run precisions were calculated by using the formula \%R.S.D. $=($ S.D. $/ M)(100)$, where $M$ is the mean of experimentally determined concentrations and S.D. is the standard deviation of M. Accuracy was calculated using the formula \%RE $=|(E-T)(100 / T)|$, where $E$ is an experimentally determined concentration and $\mathrm{T}$ is a theoretical concentration.
Recovered $\mathrm{Qu}$ and Ir were assessed at three concentration levels for plasma and at four concentration levels for urine. The analyte and IS concentrations of the replicate QC samples $(n=3)$ are given in Section 'Preparation of standard and QC solutions'. The peak areas of extracted samples with the blank biological matrices extracts fortified with the drug post extraction were compared.

The validity of the fivefold dilution procedure for the model biological samples with blank plasma was assessed by Precision and Accuracy parameters [17]. Three different concentrations of $\mathrm{Qu}$ and $\operatorname{Ir}$ (about 2500, 5000 and $10,000 \mathrm{ng} / \mathrm{ml}$ for each analyte in plasma) were evaluated by analyzing five aliquots of each concentration.

Using three and four concentration levels, each type of stability specific for bioanalytical methods was studied [17]: short-term stability, freeze-thaw stability, bench top stability, long-term stability. The analytes and IS concentrations of the replicate QC samples $(n=3)$ are given in Section 'Preparation of standard and QC solutions'. All the samples for stability studies were prepared on the basis of blank biological matrix spiked with respective amounts of the analytes in a mixture of water-AcN (3:1), containing $0.005 \%$ ascorbic acid and $0.2 \%$ L-cysteine. All samples were analyzed immediately after preparation and after the applied storage conditions. The short-term stability was examined following sample defrosting and 4-h incubation at room temperature. The freeze-thaw stability was examined after three freeze-thaw cycles.

The long-term stability was determined by analyzing three samples at each concentration level stored at $-70^{\circ}$ $\mathrm{C}$ for 108 days. The stability of the samples processed in autosampler was examined after $18 \mathrm{~h}$. Stability of the stock analytes solutions was assessed after 3 month storage and of the stock IS solution - for 22 days when kept at temperature of +2 to $+7{ }^{\circ} \mathrm{C}$.

During the development of the method, we investigated the time required to sufficiently complete enzymatic deconjugation of glucuronide and sulphate derivatives of $\mathrm{Qu}$. Meanwhile, the completeness and stability of formed aglycones were evaluated using biosamples obtained from the subjects loaded with a dose of an active substance. An enzyme solution was added to two routine samples collected $2 \mathrm{~h}$ after the administration of the formulation, and they were incubated at $37^{\circ} \mathrm{C}$ for $17 \mathrm{~h}, 20 \mathrm{~h}$ and $23 \mathrm{~h}$. The further preparation of samples and their analysis were carried out in accordance with the method which was described above.

\section{Application for pharmacokinetic study}

The pharmacokinetic study of injectable medicinal product Corvitin ${ }^{\circ}$ was carried out (March-April, 2009) in accordance with the Declaration of Helsinki, GCP and GLP requirements. The trial was registered in the Ukrainian 
national registry (Resolution of State Pharmacological Center of Ministry of Healh of Ukraine \#741/2.2.1-5 from 10.02.2009). Before the study the sponsor monitoring on GCP requirements was held, also the State Expert Center of Ministry of Health of Ukraine audits are conducted on regular basis (The audit before the trial was conducted in November, 2008). The protocol and associated informed consent forms were reviewed and approved by The Ethical Committee and the informed consent forms were signed by the volunteers prior to the study initiation. Twelve healthy volunteers (aged 18-45) years of both sexes were enrolled in the study. In order to carry out the study, each volunteer was given the formulation (combination of $\mathrm{Qu}$ and polyvinylpyrrolidone at the ratio of 1:9 in form of porous lyophilized powder of $500 \mathrm{mg}$ ), which was diluted with $50 \mathrm{ml}$ of sterile physiological solution $2 \mathrm{~h}$ before administration, by using infusion pumps ensuring the same speed of intravenous injection. The dose was calculated individually on the basis of a body surface area of volunteers at a rate of 250 $\mathrm{mg}$ of the formulation per square meter of body surface. Thus, administered doses of the product were 365 to 528 $\mathrm{mg}$ (37 to $53 \mathrm{ml}$ of the solution).

Thirteen blood samples were drawn from each subject during the study, $5.5 \mathrm{ml}$ of blood at each study time point. The blood sampling was conducted according to the following schedule: $0 \mathrm{~h}$ (pre-dose), 15, 30 and 45 min, and $1,1.5,2,4,5,6,8,12$ and $24 \mathrm{~h}$ post-dose.

The samples were put into $6 \mathrm{ml}$ plastic vacuum tubes pre-treated with sodium heparin and centrifuged within 3 min after collection. The centrifugation was conducted in the temperature-stabilized rotary chamber at $10^{\circ} \mathrm{C}$ for 5 to $6 \mathrm{~min}$ at $4000 \mathrm{~g}$. The obtained plasma was acidified ( $1 \mathrm{ml}$ of plasma was added with $0.05 \mathrm{ml}$ of $0.25 \mathrm{M}$ solution of acetic acid). The plasma was divided into two aliquots (the first one was for the analysis, the second one was for the back-up) but the volume was not less than $1.2 \mathrm{~mL}$ for each aliquot, which were transferred into the pre-labeled cryotubes. The plasma was kept at a temperature below $-70{ }^{\circ} \mathrm{C}$ at the study site and at the bioanalytical laboratory as well. The time period between blood collection and plasma transfer into $-70{ }^{\circ} \mathrm{C}$ freezer did not exceed $15 \mathrm{~min}$.

The sampling of urine was performed as follows. The volunteers were given a labelled container for urine collection for each period of time $(0-3 \mathrm{~h}, 3-6 \mathrm{~h}, 6-9 \mathrm{~h}, 9-12 \mathrm{~h}$, $12-16 \mathrm{~h}, 16-24 \mathrm{~h}$ ). The urine collection was done 'on volunteer's demand'. Before receiving an aliquot, a container was kept at temperature $+4{ }^{\circ} \mathrm{C}$. The volume of total urine obtained over the specified period was measured by using a graduated cylinder. Then the urine was mixed, and two $5 \mathrm{ml}$ aliquots were taken as soon as possible (the first aliquot was for the analysis, the second one was for the back-up), then they were immediately frozen and kept at a temperature below $-70^{\circ} \mathrm{C}$.
From the clinical site to the bioanalytical laboratory, the frozen samples were transferred in secondary containers put in cryoboxes filled with "dry ice" (solid carbon dioxide).

The developed method was used for quantification of total $\mathrm{Qu}\left(\mathrm{Qu}_{\mathrm{Tot}}\right)$, obtained after enzymatic hydrolysis, unconjugated (non-hydrolyzed) $\mathrm{Qu}\left(\mathrm{Qu}_{2}\right)$, conjugated $\mathrm{Qu}_{1}=\mathrm{Qu}_{\mathrm{Tot}}-\mathrm{Qu}_{2}$, and methyl metabolites of $\mathrm{Qu}$ with reference to Ir: total one ( $\left.\mathrm{Ir}_{\mathrm{Tot}}\right)$, obtained after enzymatic hydrolysis, unconjugated $\operatorname{Ir}\left(\operatorname{Ir}_{2}\right)$ and conjugated $\operatorname{Ir}_{1}=\operatorname{Ir}_{\mathrm{Tot}}$ - $\mathrm{Ir}_{2}$ in biological matrixes. Additionally, we determined the amounts of total bioavailable $\mathrm{Qu}$, i.e. $\mathrm{Qu}_{\mathrm{Sum}}=\mathrm{Qu}_{\mathrm{Tot}}+$ $0.95567 * \mathrm{Ir}_{\mathrm{Tot}}$ in order to assess bioavailability of $\mathrm{Qu}$ from the formulation of Corvitin .0 .95567 is a conversion factor of Ir to $\mathrm{Qu}$, taking into account the difference between their molecular masses.

The pharmacokinetic profiles were obtained for every volunteer. The findings of $\mathrm{C}_{\max }$ and $\mathrm{T}_{\max }$ were obtained by applying the common statistics.

The data analysis of pharmacokinetic parameters for $\mathrm{Qu}$ in the blood plasma was performed with the help Win-Nonlin professional software version 5.2 (Pharsight, Mountain View, CA, USA) using non-compartmental approach to the model assessment of NCA Model 202 (continuous infusion). The infusion time $15 \mathrm{~min}$ was taken into account. Area under the plasma concentration curve $\left(\mathrm{AUC}_{0-24}\right)$ values ( $\mathrm{t}$ being the time of the last plasma concentration measured) were calculated by the linear trapezoidal rule.

The AUC from 0 to infinity $\left(\mathrm{AUC}_{0-\infty}\right)$ was calculated by the software. The first-order rate constant $K_{\text {el }}$ was assessed by linear regression of time vs log of the concentration. Terminal half-life $\left(\mathrm{T}_{1 / 2}\right)$ was also calculated.

The pharmacokinetic data on determination of $\mathrm{Qu}$ in the urine were treated by using the model assessment of NCA Model 212 of the same software.

Using the least-squares method and log-transformed data of excretion rate $(R)$ of the substance in the urine, the elimination constant $\mathrm{K}_{\mathrm{el} \text {.(R) }}$ and half-life $\mathrm{T}_{1 / 2(\mathrm{R})}$ were assessed. The excretion rate of the test substance in the urine was calculated for each of $\mathrm{n}$ time periods (time of the beginning of a period $-T_{n-1}$, time of the end of a period $\left.-T_{n}\right)$ by the formula: $R=C * V /\left(T_{n}-T_{n-1}\right)$, where $\mathrm{C}$ is a concentration of analyte in the urine, in $\mathrm{ng} / \mathrm{ml}, \mathrm{V}$ is a volume of urine in the specified time period in $\mathrm{ml}$. Also the weight $(\mathrm{M})$ in $\mu \mathrm{g}$ of eliminated $\mathrm{Qu}$ within this time period in the urine was calculated: $\mathrm{M}(\mu \mathrm{g})=\mathrm{C} \times \mathrm{V} / 1000$.

It should be noted that according to the study protocol, the first time period of sampling urine was stated from 0 to $3 \mathrm{~h}$. However, the infusion of the formulation continued as per protocol for $15 \mathrm{~min}(0.25 \mathrm{~h})$. Thus, the actual first sampling period was from $0.25 \mathrm{~h}$ to $3 \mathrm{~h}$, which was taken into account in the further calculations (the mean of the first period was $1.63 \mathrm{~h}$ ). For plotting 

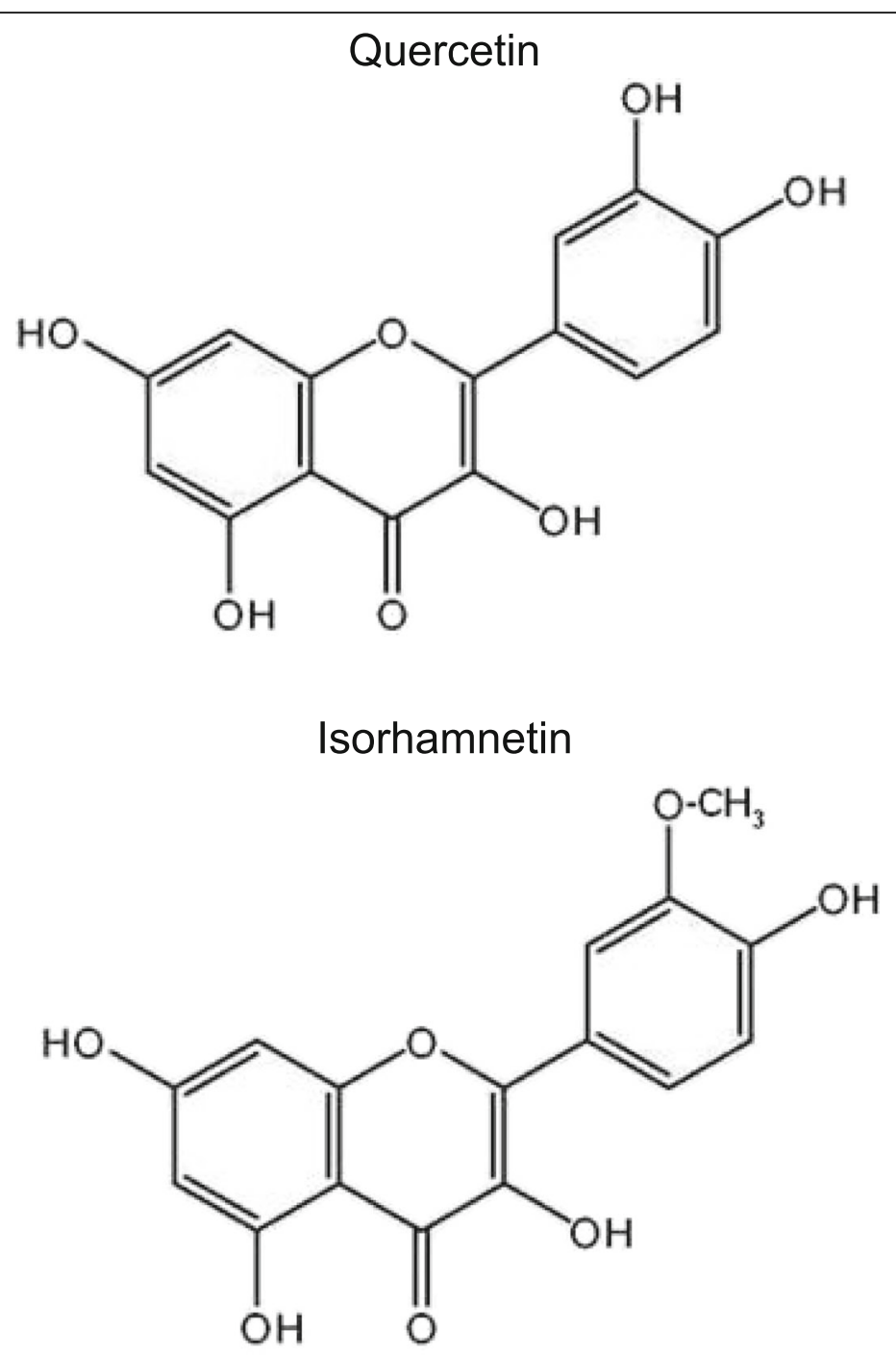

Fisetin

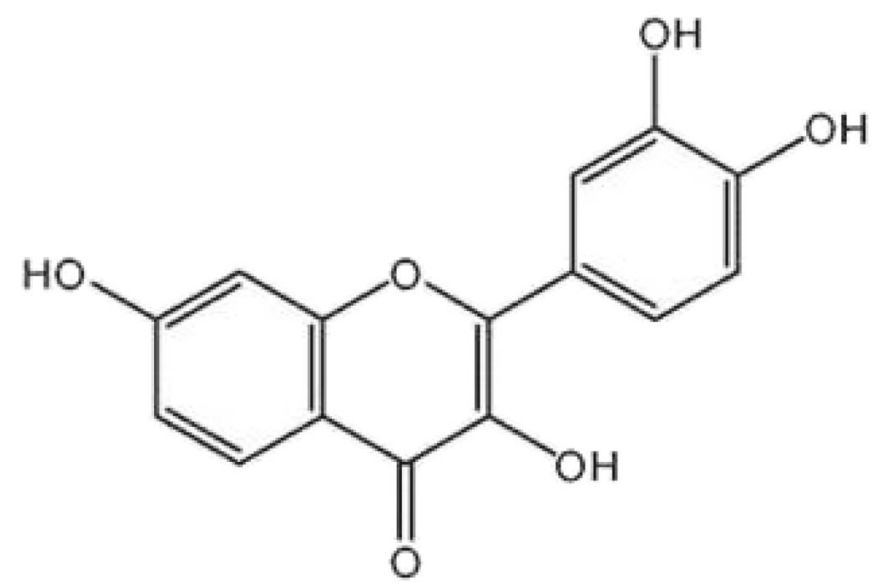

Fig. 1 Chemical structures of quercetin, its metabolite isorhamnetin and fisetin 
graphs of concentration vs time for $\mathrm{Qu}$ in urine, as well as the excretion rate of $\mathrm{Qu}$ in urine, midpoints of urine sampling periods were used.

The other pharmacokinetic parameters: $R_{\max }, T_{R \max }$, $\mathrm{AURC}_{0-\mathrm{t}}, \mathrm{AURC}_{0-\infty}$, cumulative excretion of the substance in the urine Ae, and mass of $\mathrm{Qu}$ excreted in the urine were assessed also.

\section{Results and discussion}

\section{Method development}

We used fisetin (Fs) as IS for this study. Fs (3,3',4',7-tetrahydroxyflavone) differs from $\mathrm{Qu}$ by one hydroxyl group, which is missing in position 5 . It has similar physical and chemical properties, chromatographic behaviour of the analytes, and is absent in the human plasma at the endogenous level. Fs was successfully used earlier as an IS for HPLC MS/MS determination of Qu and its sulphate and glucuronide conjugates [1]. The structural formulae of the specified substances are shown in Fig. 1.

A gradient: methanol-water solution of trifluoroacetic acid was used for chromatographic separation of glucuronides of $\mathrm{Qu}$ in paper [3]. The gradient based on acetonitrile-water solution of formic acid was used for chromatographic separation of $\mathrm{Qu}$ metabolites in the paper [5]. We used a similar to the latter gradient for chromatographic separation because a gradient elution mode demonstrated better results than an isocratic

\section{A}
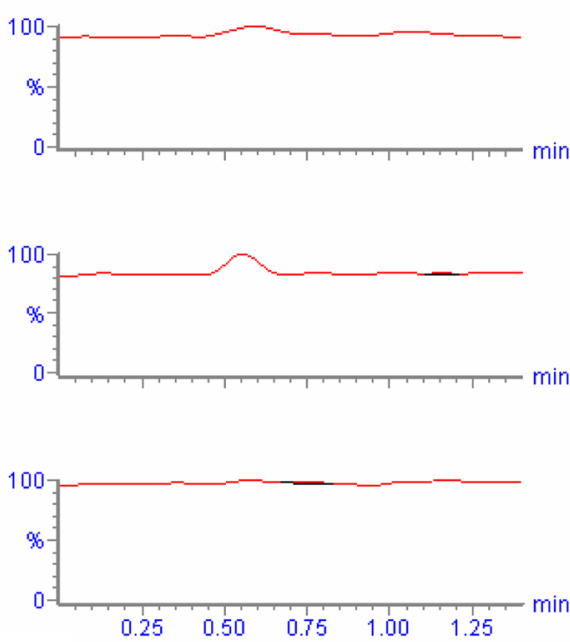

C
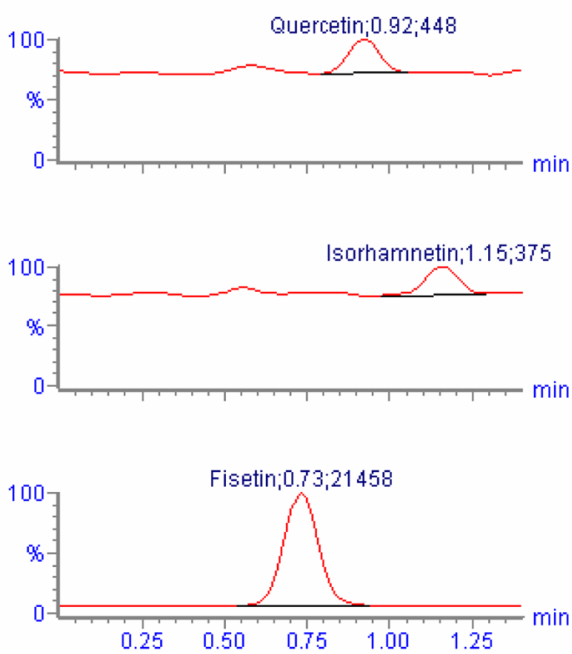

B
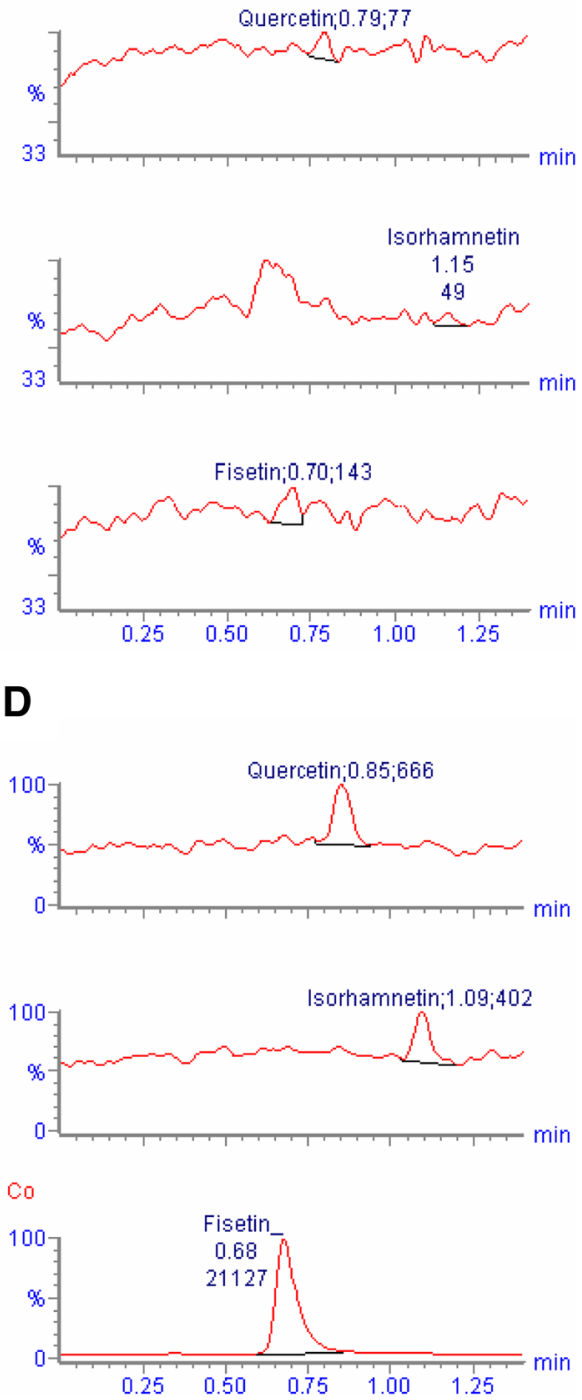

Fig. 2 Chromatograms: (a) blank plasma (Qu, Ir and IS free); (c) blank plasma spiked with $25 \mathrm{ng} / \mathrm{ml} \mathrm{Qu}$ (LLOQ) and $25 \mathrm{ng} / \mathrm{ml}$ Ir (LLOQ); (b) blank urine (Qu, Ir and IS free); (d) blank urine spiked with $100 \mathrm{ng} / \mathrm{ml} \mathrm{Qu} \mathrm{(LLOQ)} \mathrm{and} 100 \mathrm{ng} / \mathrm{ml} \mathrm{Ir}$ (LLOQ) 


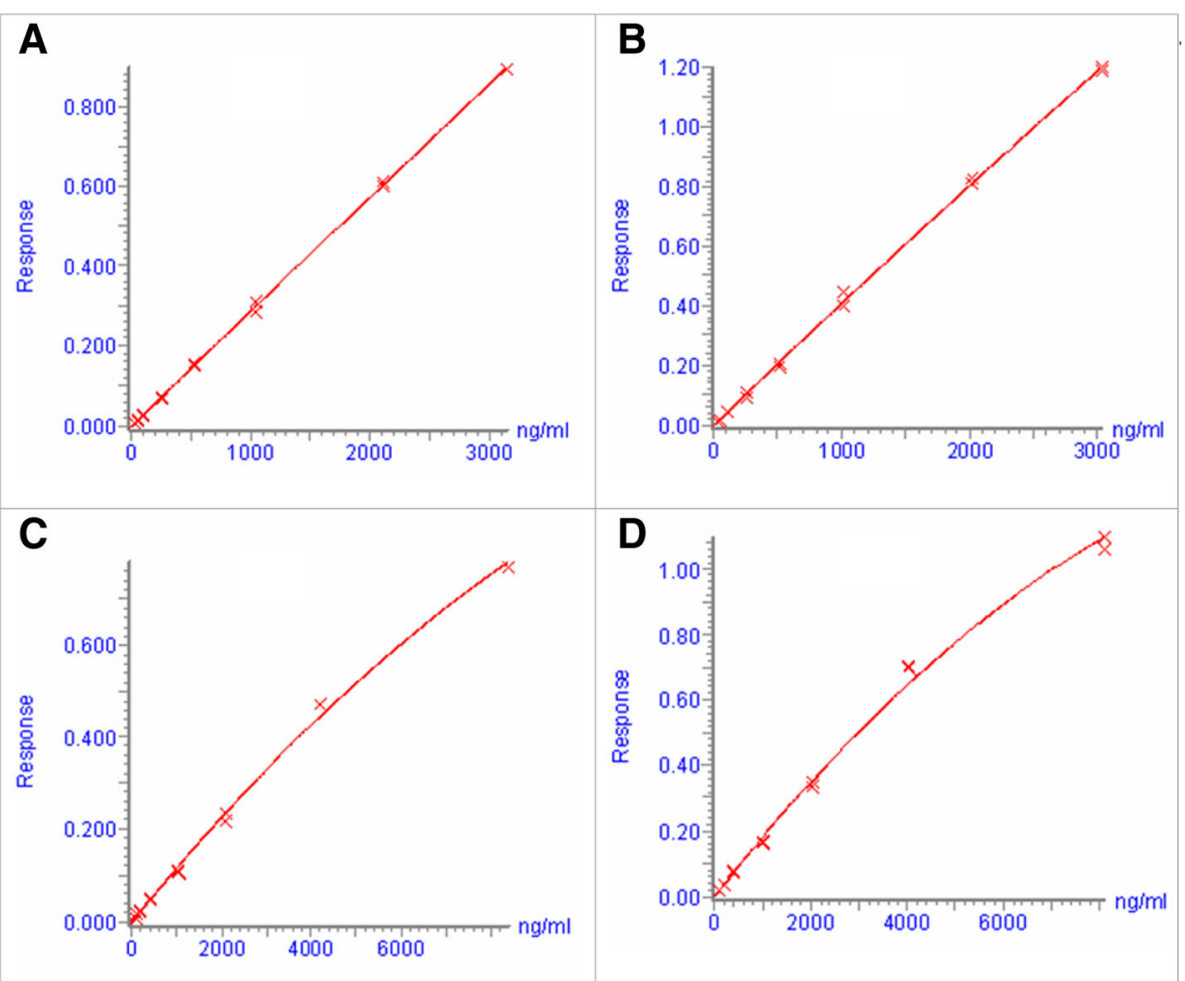

Fig. 3 Calibration curves for $\mathrm{Qu}(\mathbf{a}, \mathbf{c})$ and $\operatorname{Ir}(\mathbf{b}, \mathbf{d})$ in plasma $(\mathbf{a}, \mathbf{b})$ and urine $(\mathbf{c}, \mathbf{d})$

mode. The chromatographic conditions and parameters of detection are given in Section 'Instrumentation and methods'.

The time of data registration was $1.4 \mathrm{~min}$. A chromatographic run continued for $1.9 \mathrm{~min}$, including the time of sampling. The short time of recording chromatograms allowed injection of each test sample twice; the result was calculated as a mean of two parallel injections. The UPLC chromatography allowed significant reduction of time for recording chromatograms comparing with traditional HPLC.

The chromatograms of blank plasma, blank urine and LLOQ standards for plasma and urine are shown in Fig. 2.

$\mathrm{Qu}$ can be easily oxidized during preparation of samples, storage of the solutions, and MS-detection as mentioned in Section 'Introduction'.

To prevent oxidation of $\mathrm{Qu}$ we tried various concentrations of ascorbic acid, which was added to $\mathrm{Qu}$ and Ir solutions. Increasing concentrations of ascorbic acid, however, did not give any positive effect to stabilize of $\mathrm{Qu}$. At the same time using L-cysteine mixed with ascorbic acid as antioxidant led to the stabilization of $\mathrm{Qu}$ and Ir both during handling of samples and chromatographic runs with mass detection. Higher efficiency of L-cysteine as antioxidant can be explained by greater negative reduction potential of the cysteine/cystine system then in the ascorbic acid/dehydroascorbic acid pair
[18]. Antioxidant concentrations used at each step of handling of samples are given in Section 'Preparation of standard and QC solutions'. According to our data, the effective method proposed to stabilize of $\mathrm{Qu}$ and Ir in solutions was used for the first time.

We used a procedure of solid phase extraction by using Oasis ${ }^{\circ}$ HLB cartridge after pre-dilution of samples with $0.5 \mathrm{M}$ solution of phosphoric acid [19] to improve the separation of the analytes.

As mentioned in Section 'Introduction' investigation of pharmacokinetics of $\mathrm{Qu}$ products requires determination of both free $\mathrm{Qu}$ and its metabolites - methylated derivatives and sulphate and glucuronic conjugates.

In order to assay total glucuronide and sulphate metabolites they were enzymatically deconjugated.

Table 1 The coefficients of the regression equation of calibration curve $\left(Y=a \cdot X^{2}+b \cdot X+c\right)$ and coefficients of determination

\begin{tabular}{|c|c|c|c|c|}
\hline \multirow{2}{*}{$\begin{array}{l}\text { Component } \\
\text { to be } \\
\text { determined/ } \\
\text { biomatrix }\end{array}$} & \multicolumn{3}{|c|}{ Coefficients of regression equation } & \multirow{2}{*}{$\begin{array}{l}\text { Coefficients of } \\
\text { determination } \\
R^{2}\end{array}$} \\
\hline & $A$ & $b$ & c & \\
\hline Qu/plasma & $-1.390^{*} 10^{-8}$ & 0.0003208 & -0.00139 & 0.9985 \\
\hline Ir/plasma & $-8.119^{*} 10^{-9}$ & 0.0002572 & -0.00262 & 0.9937 \\
\hline Qu/urine & $8.866^{*} 10^{-10}$ & 0.0000523 & 0.00106 & 0.9984 \\
\hline Ir/urine & $1.766^{*} 10^{-9}$ & 0.0000978 & 0.00406 & 0.9983 \\
\hline
\end{tabular}


Since methylated $\mathrm{Qu}$ derivatives are resistant to enzymes, calibration curves were built with Ir standards for quantification of this kind of compounds.

It was found that enzymatic deconjugation of $\mathrm{Qu}$ and methylquercetin derivatives lasted for $17 \mathrm{~h}$ and this time interval was used in all the further experiments for deconjugation.

\section{Results of method validation}

It was established that LLOQ of $\mathrm{Qu}$ and Ir were $25 \mathrm{ng} / \mathrm{ml}$ in blood plasma and $100 \mathrm{ng} / \mathrm{ml}$ in urine for each analyte. At that the signal/noise ratios in plasma were: for $\mathrm{Qu}>8.2$, for Ir > 7.6; in urine: for $\mathrm{Qu}>45.4$, for Ir > 100.4. These results meet the acceptance criteria of $\geq 5$ [17]. Qu and Ir concentrations at the LLOQ level were determined at accuracy $(\mathrm{RE}<14 \%)$ and precision $(\mathrm{RSD}<17 \%)$, which meet the established criteria [17] for both parameters $(\leq 20 \%)$.

The method selectivity was demonstrated by using 3 different batches of respective blank biological matrices spiked with Qu and Ir at LLOQ amounts, and with IS Fs with appropriate concentration. The ratios of peak areas in the chromatograms of solutions of blank biological matrix and solutions for determining selectivity were the following: for plasma samples: $\mathrm{Qu}<0.161$, $\mathrm{Ir}<0.149$; for
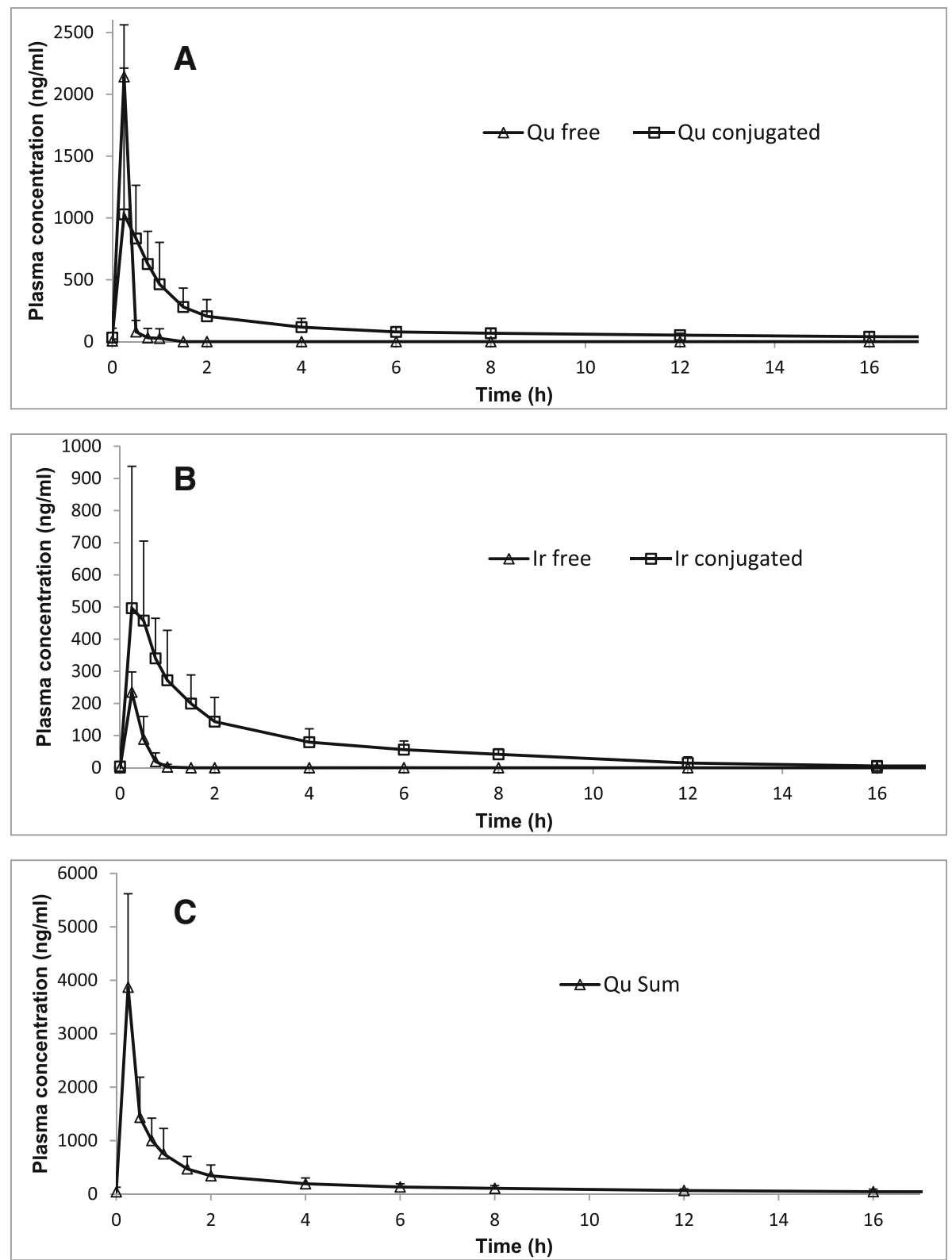

Fig. 4 Mean plasma concentration - time profile following intravenous administration of $250 \mathrm{mg} / \mathrm{m}^{2}$ of human body surface area. a - free Qu and conjugated metabolites of Qu, $\mathbf{b}$ - free Ir and conjugated metabolites of Ir, c - sum of free and conjugated metabolites of Qu and Ir (+ S.D.) 
Table 2 Mean pharmacokinetic parameters of Qu in plasma for 12 volunteers after intravenous administration of Corvitin ${ }^{\circledR}$ (average dose of anhydrous Qu was $44 \mathrm{mg}$ )

\begin{tabular}{llllll}
\hline Parameters & Qu free & Qu conjug & Ir free & Ir conjug & Qu sum \\
\hline$C_{\max }(\mathrm{ng} / \mathrm{ml})$ & $2142 \pm 419$ & $1400 \pm 941$ & $252 \pm 69$ & $657 \pm 359$ & $3871 \pm 1748$ \\
$T_{\max }(\mathrm{h})$ & $0.25 \pm 0.00$ & $0.42 \pm 0.12$ & $0.27 \pm 0.07$ & $0.46 \pm 0.14$ & $0.25 \pm 0.00$ \\
$A \cup C_{0-24}(\mathrm{~h} \cdot \mathrm{ng} / \mathrm{ml})$ & $545 \pm 142$ & $2344 \pm 1251$ & $83.24 \pm 38.54$ & $1155 \pm 604$ & $4137 \pm 1796$ \\
$A \cup C_{0-\infty}(\mathrm{h} \cdot \mathrm{ng} / \mathrm{ml})$ & $583.0 \pm 91.8$ & $2839 \pm 1523$ & $132.4 \pm 28.1$ & $1362 \pm 670$ & $4596 \pm 2107$ \\
$A \cup C_{0-24} / A \cup C_{0-\infty}$ (per cent) & $95.1 \pm 6.0$ & $82.9 \pm 5.3$ & $92.5 \pm 4.0$ & $84.3 \pm 4.1$ & $90.9 \pm 3.6$ \\
$K_{\mathrm{el}}$ & $6.08 \pm 2.47$ & $0.11 \pm 0.05$ & $4.02 \pm 0.68$ & $0.19 \pm 0.07$ & $0.13 \pm 0.03$ \\
$T_{1 / 2}(\mathrm{~h})$ & $0.13 \pm 0.06$ & $6.90 \pm 2.41$ & $0.18 \pm 0.03$ & $4.40 \pm 2.09$ & $5.80 \pm 1.45$ \\
\hline
\end{tabular}

Mean \pm S.D., $n=12$

urine samples: $\mathrm{Qu}<0.259$, Ir $<0.224$, which meet the criteria established by our laboratory $(<0.5)$.

The calibration curves for plasma and urine are shown in Fig. 3. The results of blank and zero samples were not taken into consideration when constructing the calibration function. The most adequately all the calibration curves are described by using a quadratic model with the weighting factor of $1 / \mathrm{X}$ (i.e., $1 /$ concentration).

The values of coefficients of the regression equation of the calibration curve $\mathrm{Y}=\mathrm{a} \cdot \mathrm{X}^{2}+\mathrm{b} \cdot \mathrm{X}+\mathrm{c}$ that were determined during the method validation of assay of $\mathrm{Qu}$ and Ir in plasma and urine are given in Table. 1.

The table shows that the coefficients of determination $\left(R^{2}\right)$ were greater than 0.990 in all the determinations. The back calculated concentrations of the calibration standards complied with the acceptance criteria [17].

When assessing the parameters Precision and Accuracy for quantification of $\mathrm{Qu}$ and Ir in plasma, it was established that the R.S.D. (both intra- and inter-run) for $\mathrm{Qu}$ was not greater than $14.5 \%$, and for $\mathrm{Ir}-11.6 \%$ at the value of accuracy for $\mathrm{Qu}$ and Ir not greater than 6.8 and 9.7\%, respectively. It was established that R.S.D. (both intra- and inter-run) for $\mathrm{Qu}$ was not greater than 9.4\%, and for Ir $-6.9 \%$ at the value of accuracy for Qu and Ir not greater than 7.5 and $6.5 \%$, respectively for quantification of $\mathrm{Qu}$ and $\mathrm{Ir}$ in urine.

The recovery values of $\mathrm{Qu}$ and Ir from plasma were of $61.8-65.5 \%$ and of $73.5-76.9 \%$, respectively. The recovery values of $\mathrm{Qu}$ and Ir from urine were of $68.5-73.3 \%$ and of $75.1-77.4 \%$, respectively.

The level of expected concentrations after intravenous administration of $\mathrm{Qu}$ was a priori rather complicated to establish. The procedure of fivefold dilution of biosamples with blank plasma was validated in order to prepare a routine analysis. Validation was performed according to Precision and Accuracy parameters. The obtained results of assessment of these parameters were as follows: R.S.D. for both analytes was not greater than $6.3 \%$, RE for $\mathrm{Qu}$ was less than
14.4 , and for $\operatorname{Ir}<14.8 \%$, which confirmed the validity of the dilution procedure [17].

The stability study was performed using model biosamples containing $\mathrm{Qu}$ and $\mathrm{Ir}$ in respective blank biological matrix. It was established that analytes are stable after undergoing three freeze-thaw cycles, as well as after defrosting the samples and keeping them at room temperature for $4 \mathrm{~h}$. Additionally, it was shown that analytes are stable when the abovementioned biosamples were stored at the temperature below minus $70^{\circ} \mathrm{C}$ for 108 days. Storage time of the real biosamples did not exceed 103 days.

The stability of solutions in an autosampler for $18 \mathrm{~h}$ was also demonstrated.

The mean RE values for all the concentration levels and above listed stabilities tested were not greater than $15 \%$, i.e. they met the established acceptance criteria [17]. The stability of the stock solutions of analytes for 3 months, and IS solutions for 22 days that were stored at the temperature of +2 to $+7{ }^{\circ} \mathrm{C}$ was also demonstrated with $\mathrm{RE}<3.7 \%$.

\section{Pharmacokinetic study}

Figure 4 shows the mean plasma concentration/time profiles of free $\mathrm{Qu}$ and conjugated metabolites of $\mathrm{Qu}$,

Table 3 Mean pharmacokinetic parameters of Qu in urine for 12 volunteers after intravenous administration of Corvitin ${ }^{\oplus}$ (average dose of anhydrous Qu was $44 \mathrm{mg}$ )

\begin{tabular}{llll}
\hline Parameters & Qu conjug & Ir conjug & Qu sum \\
\hline$R_{\max }(\mu \mathrm{g} / \mathrm{h})$ & $985.3 \pm 292.5$ & $421.8 \pm 129.1$ & $1388 \pm 405$ \\
$T_{\text {Rmax }}(\mathrm{h})$ & $1.63 \pm 0.00$ & $1.63 \pm 0.00$ & $1.63 \pm 0.00$ \\
AURC $_{0-\mathrm{t}}(\mu \mathrm{g})$ & $2814 \pm 969$ & $1392 \pm 520$ & $4169 \pm 1435$ \\
AURC $_{0-\infty}(\mathrm{ng})$ & $2769 \pm 988$ & $1482 \pm 557$ & $4123 \pm 1492$ \\
$A \cup R C_{0-\mathrm{t}} / A \cup C_{0-\infty}$ (per cent) & $98.98 \pm 0.28$ & $97.12 \pm 3.06$ & $99.09 \pm 0.52$ \\
Ae (ng) & $3164 \pm 868$ & $1548 \pm 414$ & $4643 \pm 1231$ \\
$K_{\mathrm{el}(\mathrm{R})}(1 / \mathrm{h})$ & $0.35 \pm 0.21$ & $0.43 \pm 0.15$ & $0.35 \pm 0.17$ \\
$T_{1 / 2}(\mathrm{~h})$ & $2.60 \pm 1.26$ & $1.81 \pm 0.71$ & $2.33 \pm 0.84$ \\
\hline
\end{tabular}

Mean \pm S.D., $n=12$ 


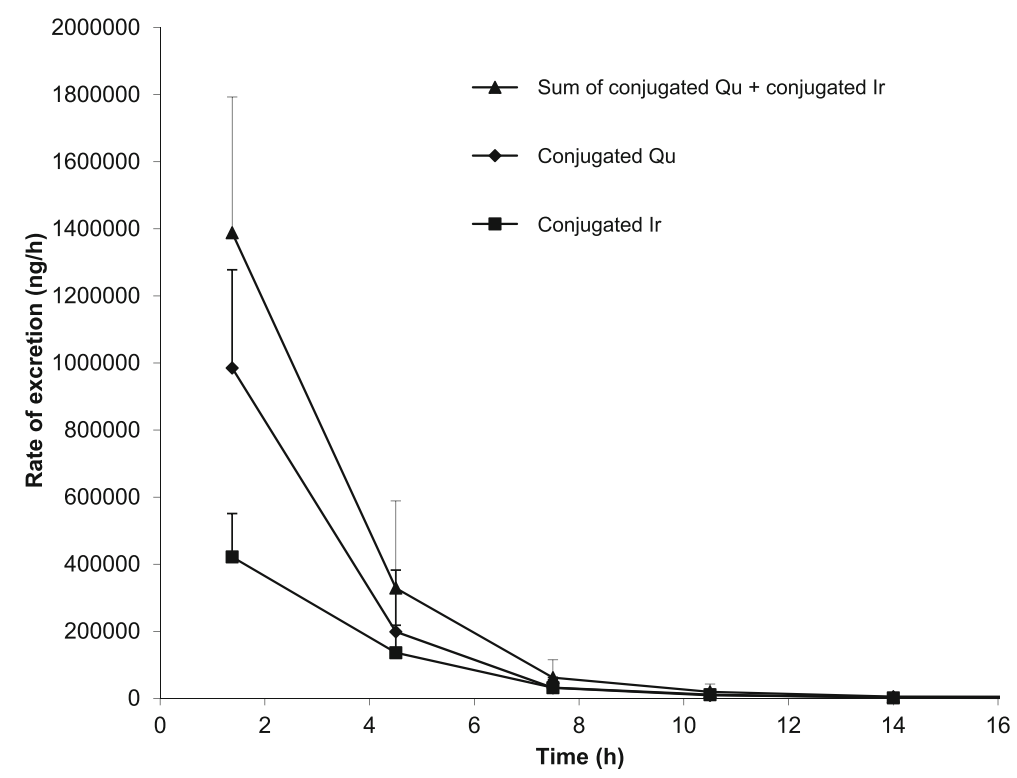

Fig. 5 Mean rate of Qu excretion in urine - time profiles following intravenous administration of a dose with reference to $250 \mathrm{mg} / \mathrm{m}^{2}$ of human body surface area for conjugated metabolites of Qu, conjugated metabolites of Ir and for total conjugated metabolites of Qu and Ir

free Ir and conjugated metabolites of Ir, and summarised: free $\mathrm{Qu}$ and $\mathrm{Ir}$ and conjugated metabolites of $\mathrm{Qu}$ and Ir.

The basic pharmacokinetic parameters of these curves are shown in Table 2. The table shows that $\mathrm{Qu}$ is rapidly metabolized upon administration (the infusion time was $15 \mathrm{~min}$, this was the first time point to sample) to form both glucoronide and sulphate conjugates, and, in a less degree, methyl metabolites and their conjugates. Over this time about $45 \%$ of $\mathrm{Qu}$ is metabolized; at that, about $28 \%$ of $\mathrm{Qu}$ forms sulphate and glucuronide metabolites, about 5\% turns into metabolites being methylated $\mathrm{Qu}$, and $12 \%$ - into sulphate and glucuronide conjugates of methylated metabolites.

Free $\mathrm{Qu}$ was not detected in plasma of any volunteer in $1.5 \mathrm{~h}$ after infusion. $\mathrm{T}_{1 / 2}$ of free $\mathrm{Qu}$ and free $\mathrm{Ir}$ are 0.13 and $0.18 \mathrm{~h}$, respectively, which indicates a very rapid metabolism of aglycones, which also is confirmed by high elimination constants of these substances $\left(6.08 \mathrm{~h}^{-1}\right.$ and $4.02 \mathrm{~h}^{-1}$, respectively). At the same time, the parameter $\mathrm{T}_{1 / 2}$ for conjugated $\mathrm{Qu}$, conjugated $\mathrm{Ir}$, and total $\mathrm{Qu}$ are $6.90,4.40$ and $5.80 \mathrm{~h}$, respectively, indicating their relatively slow excretion from the body (elimination constants of these compounds are $0.11,0.19$ and 0.13 , respectively). The highest value of $\mathrm{C}_{\max }$ was observed for the total $\mathrm{Qu}$ at $\mathrm{T}_{\max }=0.25 \mathrm{~h}$, i.e. immediately after infusion that is natural for injectable drugs.

When determining $\mathrm{Qu}$ and its methyl metabolites in terms of Ir in urine, it was found that free $\mathrm{Qu}$ and $\mathrm{Ir}$ in urine were absent at all time points. This is natural, taking into account the rapid metabolism of these compounds to the sulphate form and glucuronide conjugates. Figure 5 shows the elimination rate/time curves for conjugated $\mathrm{Qu}$, conjugated Ir, and total Qu. Table 3 demonstrates the basic elimination pharmacokinetic parameters of these substances in urine. For the points where the volume of urine samples was equal to zero and which were not end points, the mean elimination rate equal to the mean elimination rate at the previous and following time points was taken. The cumulative weight of total $\mathrm{Qu}$ eliminated in urine over $24 \mathrm{~h}$ was $4.57 \mu \mathrm{g}$, which is about $10.2 \%$ of the infused Qu.

Thus, the obtained data on the pharmacokinetics of Corvitin represent the full characteristic behaviour of $\mathrm{Qu}$ and its metabolites in the human blood beginning from its intravenous administration.

\section{Conclusion}

We developed a new quantification method for quercetin and its metabolites in plasma and urine by using L-cysteine as an antioxidant. The method is based on the solid-phase extraction of quercetin and products of its enzymatic hydrolysis followed by the analysis of an extract by the UPLC-MS/MS method. The method was successfully applied in the pharmacokinetic study of the parenteral formulation of quercetin used in medical practice.

\section{Acknowledgements}

We thank to SIC 'Borshchahivskiy Chemical-Pharmaceutical Plant CJSC', Ukraine for funding the study.

\section{Funding}

SIC 'Borshchahivskiy Chemical-Pharmaceutical Plant CJSC', Ukraine. 
Availability of data and materials

Not applicable.

\section{Authors' contributions}

All authors read and approved the final manuscript.

\section{Ethics approval and consent to participate}

The study was approved by the Central Ethics Committee of Ministry of Health of Ukraine (resolution \#5.12-194/KE from 04.03.2099) and by the Local Ethics Committee (resolution \#01/19 from 10.03.2009) with minor amendments to the trial protocol (resolution \#02/19 from 27.03.2009).

\section{Consent for publication}

Not applicable.

\section{Competing interests}

Not applicable.

\section{Publisher's Note}

Springer Nature remains neutral with regard to jurisdictional claims in published maps and institutional affiliations.

\section{Author details}

${ }^{1}$ National University of Pharmacy, 27 Pushkinska Street, Kharkiv 61057 Ukraine. ${ }^{2}$ Bioanalytical laboratory Clinfarm, 3 Shevchenko Street, Irpin, Kiev Oblast 08200, Ukraine.

Received: 5 October 2018 Accepted: 24 January 2019

Published online: 31 January 2019

\section{References}

1. Wang L, Morris ME. Liquid chromatography-tandem mass spectroscopy assay for quercetin and conjugated quercetin metabolites in human plasma and urine. J Chromatogr B. 2005;821:194-201.

2. Day AJ, Mellon F, Barron D, Sarrazin G, Morgan MRA, Williamson G. Human metabolism of dietary flavonoids: identification of plasma metabolites of quercetin. Free Radic Res. 2001:35:941-52.

3. Wittig J, Herderich M, Graefe EU, Veit M. Identification of quercetin glucuronides in human plasma by high-performance liquid chromatographytandem mass spectrometry. J Chromatogr B. 2001;753:237-43.

4. Hong YJ, Mitchell AE. Metabolic profiling of flavonol metabolites in human urine by liquid chromatography and tandem mass spectrometry. J Agric Food Chem. 2004;52:6794-801.

5. Manach C, Morand C, Crespy V, Demigne C, Texier O, al e. Quercetin is recovered in human plasma as conjugated derivatives which retain antioxidant properties. FEBS Lett. 1998:426:331-6.

6. Justino GC, Santos MR, Canario S, Borges C, Florencio MH, al e. Plasma quercetin metabolites: structure-antioxidant activity relationships. Arch Biochem Biophys. 2004;432:109-21.

7. Larson AJ, Symons JD, Jalili T. Quercetin: a treatment for hypertension? - a review of efficacy and mechanisms. Pharmaceuticals. 2010;3:237-50.

8. Manach C, Williamson G, Morand C, Scalbert A, Remesy C. Bioavailability and bioefficacy of polyphenols in humans. I. Review of 97 bioavailability studies. Am J Clin Nutr. 2005;81:230S-42S.

9. Lodi F, Jimenez R, Moreno L, Kroon PA, Needs PW, al e. Glucuronidated and sulfated metabolites of the flavonoid quercetin prevent endothelial dysfunction but lack direct vasorelaxant effects in rat aorta. Atherosclerosis. 2009;204:34-9.

10. Boesch-Saadatmandi C, Loboda A, Wagner AE, Stachurska A, Jozkowicz A, al e. Effect of quercetin and its metabolites isorhamnetin and quercetin-3glucuronide on inflammatory gene expression: role of miR-155. J Nutr Biochem. 2011;22:293-9.

11. Manach C, Morand C, Demigne C, Texier O, Regerat F, Remesy C, al e. Bioavailability of rutin and quercetin in rats. FEBS Lett. 1997:409:12-6.

12. Mullen W, Boitier A, Stewart AJ, Crozier A. Flavonoid metabolites in human plasma and urine after the consumption of red onions: analysis by liquid chromatography with photodiode array and full scan tandem mass spectrometric detection. J Chromatogr A. 2004;1058:163-8.

13. Egert S, Wolffram S, Bosy-Westphal A, Boesch-Saadatmandi C, Wagner AE, al e. Daily quercetin supplementation dose-dependently increases plasma quercetin concentrations in healthy humans. J Nutr. 2008;138:1615-21.
14. Yang CY, Hsiu SL, Wen KC, Lin SP, Tsai SY, al e. Bioavailability and metabolic pharmacokinetics of Rutin and quercetin in rats. J Food Drug Anal. 2005;13:244-50

15. Gugler R, Leschik M, Dengler HJ. Disposition of quercetin in man after single oral and intravenous dose. Eur J Clin Pharmacol. 1975:9:229-34.

16. Ferry DR, Smith A, Malkhandi J, Fyfe DW, deTakats PG, al e. Phase I clinical trial of the flavonoid quercetin: pharmacokinetics and evidence for in Vivo tyrosine kinase inhibition. Clin. Cancer Res. 1996;2:659-68.

17. U.S. Department of Health and Human Services, Food and Drug Administration Centre for Drug Evaluation and Research (CDER), Centre for Veterinary Medicine (CVM) (May 2001). Guidance for Industry: Bioanalytical Method Validation.

18. Burton K (1961) Biochemists' Handbook. E and FN Spon Ltd., London, 90.

19. Ishii K, Furuta T, Kasuya Y. High-performance liquid chromatographic determination of quercetin in human plasma and urine utilizing solid-phase extraction and ultraviolet detection. J Chromatogr B. 2003;794:49-56.

\section{Submit your manuscript to a SpringerOpen ${ }^{\circ}$ journal and benefit from:}

- Convenient online submission

- Rigorous peer review

- Open access: articles freely available online

- High visibility within the field

- Retaining the copyright to your article

Submit your next manuscript at $\boldsymbol{\nabla}$ springeropen.com 